\title{
Strain Sensor Based on Embedded Fiber Bragg Grating in Thermoplastic Polyurethane Using the 3D Printing Technology for Improved Sensitivity
}

\author{
Harith AHMAD ${ }^{1,2}$, Mohamad Ashraff ALIAS ${ }^{1}$, Mohammad Faizal ISMAIL ${ }^{1 *}$, \\ Nor Najwa ISMAIL ${ }^{1}$, Muhammad Khairol Annuar ZAINI ${ }^{1}$, Kok Sing LIM ${ }^{1}$, \\ Gilberto BRAMBILLA ${ }^{3}$, Kenneth T. V. GRATTAN ${ }^{4}$, and B. M. Azizur RAHMAN ${ }^{4}$ \\ ${ }^{1}$ Photonics Research Centre, University of Malaya, Kuala Lumpur 50603, Malaysia \\ ${ }^{2}$ Department of Physics, Faculty of Science, University of Malaya, Kuala Lumpur 50603, Malaysia \\ ${ }^{3}$ Optoelectronics Research Centre, University of Southampton, Southampton SO17 1BJ, UK \\ ${ }^{4}$ School of Mathematics, Computer Science and Engineering, City University of London, London EC1V OHB, UK \\ ${ }^{*}$ Corresponding author: Mohammad Faizal ISMAIL_ E-mail: faizalis@um.edu.my
}

\begin{abstract}
A new and easy-to-fabricate strain sensor has been developed, based on fiber Bragg grating (FBG) technology embedded into a thermoplastic polyurethane filament using a 3-dimensional (3D) printer. Taking advantage of the flexibility and elastic properties of the thermoplastic polyurethane material, the embedding of the FBG provides durable protection with enhanced flexibility and sensitivity, as compared to the use of a bare FBG. Results of an evaluation of its performance have shown that the FBG sensors embedded in this way can be applied effectively in the measurement of strain, with an average wavelength responsivity of $0.0135 \mathrm{~nm} / \mathrm{cm}$ of displacement for tensile strain and $-0.0142 \mathrm{~nm} / \mathrm{cm}$ for compressive strain, both showing a linearity value of up to $99 \%$. Furthermore, such an embedded FBG-based strain sensor has a sensitivity of $\sim 1.74$ times greater than that of a bare FBG used for strain measurement and is well protected and suitable for in-the-field use. It is also observed that the thermoplastic polyurethane based (TPU-based) FBG strain sensor carries a sensitivity value of $\sim 2.05$ times higher than that of the polylactic acid based (PLA-based) FBG strain sensor proving that TPU material can be made as the material of choice as a "sensing" pad for the FBG.
\end{abstract}

Keywords: Fiber-optic sensors; fiber Bragg grating; 3D printing technology; multiple-point strain measurement

Citation: Harith AHMAD, Mohamad Ashraff ALIAS, Mohammad Faizal ISMAIL, Nor Najwa ISMAIL, Muhammad Khairol Annuar ZAINI, Kok Sing LIM, et al., "Strain Sensor Based on Embedded Fiber Bragg Grating in Thermoplastic Polyurethane Using the 3D Printing Technology for Improved Sensitivity,” Photonic Sensors, 2022, 12(3): 220302.

\section{Introduction}

The optical fiber sensing technology has been applied in a wide range of applications due to its adaptability in different types of harsh environments and possessing several other beneficial attributes.
The emergence of optical fiber sensors has created an effective, and indeed improved group of alternatives to existing technologies for many in-the-field measurement situations, as it is immune to electromagnetic interference (EMI) [1], has better resistance to corrosion [2], offers easy installation [3]

Received: 25 May 2021 / Revised: 13 October 2021

(C) The Author(s) 2022. This article is published with open access at Springerlink.com

DOI: 10.1007/s13320-021-0646-1

Article type: Regular 
and provides excellent geometric adaptability [4]. As fiber Bragg grating (FBG) is one of the most common types of fiber optic sensor technology, it possesses all of these advantages compared to other conventional sensors such as electrical and piezoelectric sensors [5]. Over time, these different FBG-based sensors have been applied across various fields due to their being small in size and lightweight [6], showing good mechanical flexibility [7], compatibility with quasi-distributed monitoring systems [8], and allowing a variety of multiplexing arrangements [9], as well as continuous monitoring [10], good chemical stability, and potentially reducing costs and time needed for the whole measurement process [11]. The advantages of this technology have been proven in the wide use of FBG-based sensors for automated long-distance and remote monitoring continuously $[12,13]$.

This paper builds on the previous expertise of the authors and results reported in other prior works. The FBG forming the basis of the sensor has been coated using various materials, such as epoxy resins [12] and ribbon tapes [14] for protection. For example, Tanaka et al. [15] have developed an FBG-based strain sensor embedded in composite laminates, which comprises carbon fiber reinforced plastic (CFRP) and epoxy resin and is used for temperature-compensated strain measurement. Alternatively, Chen et al. [16] have implemented a different approach in strain sensing in which the FBG sensors are directly embedded into the CFRP, without any protective casing between the fiber and composite sheets in which, however, a complex response is obtained. A disadvantage is that these methods are time-consuming as some require different molds and special tools and show lower sensor performance repeatability. However, the rapid advancement of the 3-dimensional (3D) printing technology can be brought to bear on this problem and has been implemented to allow the FBGs forming the sensor system to be embedded, as this allows a high level of flexibility in the manufacturing process [17], rapid design, and consequent production: this making the sensor solution more cost-effective, as well as minimizing waste and thus importantly offering a more environmentally friendly solution [18-20] to key measuring problems.

There is a broad range of $3 \mathrm{D}$ printing filaments available, such as polylactic acid (PLA) [19], acrylonitrile butadiene styrene (ABS) [18], and thermoplastic polyurethane (TPU) [6] that can be used and chosen according to the functionalities and dimensions of the printed end product required, recognizing that different filaments have different properties. In contrast to the traditional processing and manufacturing technology used, in which raw materials are processed to produce end products, the $3 \mathrm{D}$ printing technology is an aspect of additive manufacturing, in which the raw materials are stacked layer-by-layer to "print" the end product and can be very useful in optical sensors [19]. An example of it is FBG sensor which is small in size [13] and lightweight [21], and has good compatibility with a wide range of substrates [4]. They can easily be embedded in a 3D-printed structure to perform a variety of real-time measurements, such as monitoring the strains and stresses developing within it [22]. One of the pioneering works using this method was proposed by Hong et al. [1], who have developed an FBG-based tilt sensor in which the FBG was embedded in a polylactic acid (PLA) material by using the fused deposition modelling process (FDM) [7] and applied to measure both tension and compressive strain that resulted from using the device at different tilt angles. It was found that this FBG-based tilt sensor showed a variety of advantages, especially when used in strain measurements inside a pipe or tubing, as it has high sensitivity, ease of fabrication, and fits well with rapid prototyping.

Furthermore, Lee et al. [23] have proposed in which a temperature sensor was developed based on mechanically-induced long-period fiber gratings. 
This temperature sensor was placed on a 3D printed V-grooved plate consisting of thermoplastic in a special assembly casing made of ABS. It operated based on the linear change of both resonance wavelength and loss, depending on the environmental temperature. Another work by Liveira et al. [24] have also demonstrated the use of long-period gratings (LPG) to fabricate a strain and torsion sensitive fiber optic sensor, which was achieved through the epoxy bonding of the optical sensor onto the grooved plate, which was 3D printed using materials made of polymer resin. This was accomplished through the liquid crystal display (LCD) shadow mask technique based on a 3D printing method known as digital light processing (DLP). Finally, Zhang et al. [19] have also implemented the 3D printing technology in this context, where an FBG-based pressure sensor was developed and embedded inside a PLA material, then used for measurements of vertical pressure.

However, despite these reports, PLA-based sensors have their drawbacks when used for measuring the magnitude of strain, especially in circular structures, as their rigidity limits the stretchability of the material [25]. Consequently, more stretchable and flexible materials are needed for the embedment of the FBG. To address it in this work, a simple yet compact embedded FBG-based strain sensor system is discussed, which is easily fabricated using a 3D printer that uses a thermoplastic polyurethane (TPU) filament. The TPU is used to embed the FBGs, due to its high flexibility and durability [19], enabling it to withstand much higher tensile and compressive forces, as compared to its more common counterparts, PLA, and ABS (which are harder and less flexible [18]). Another advantage of the TPU is that it has a higher strain sensitivity than PLA, ABS, and other types of filament, which importantly makes it applicable in the measurement of strains on a non-flat or circular material [11] such as pipes. By this, in-the-field measurements for structural monitoring can be made possible for industries requiring strain measurements to get the strain profile for a clearer view. This will make TPU a good sensing pad for applications such as landslide monitoring or structural health monitoring as it will provide a greater strain representation and profile. In addition, a commercial adhesive material can be used to bond the FBG and the inner walls of the TPU material as it has good sensitivity coefficients and can function well over a wide range of temperatures [26].

\section{Sensor structure and its operational mechanism}

\subsection{FBG-based strain sensor design}

The embedded FBG-based strain sensor discussed in this work is shown in Fig. 1, which shows an FBG sensor embedded inside a 3D-printed TPU material. In this design, the FBG was embedded in a cuboid-shaped formation, with a dimension of $40 \mathrm{~mm} \times 1.8 \mathrm{~mm} \times 1.8 \mathrm{~mm}$ and an infill density of $20 \%$. The layer thickness was set to be at $0.1 \mathrm{~mm}$ to ensure a smooth surface of the printed structure and ensure a good vertical resolution during printing. Furthermore, the printing speed was adjusted to $40 \mathrm{~mm} / \mathrm{s}$ due to the material properties of TPU, as set by the software of the $3 \mathrm{D}$ printer (Cura software), which was discussed in $[18,25]$. As shown in Fig. 1, the bottom of the sensor has been designed and printed as a thin base or raft to allow better bed adhesion to the measured structure. The sensor has been constructed so that the FBG was positioned through the holes printed $(1.8 \mathrm{~mm} \times$ $1.8 \mathrm{~mm}$ ) and pre-stressed before being glued using

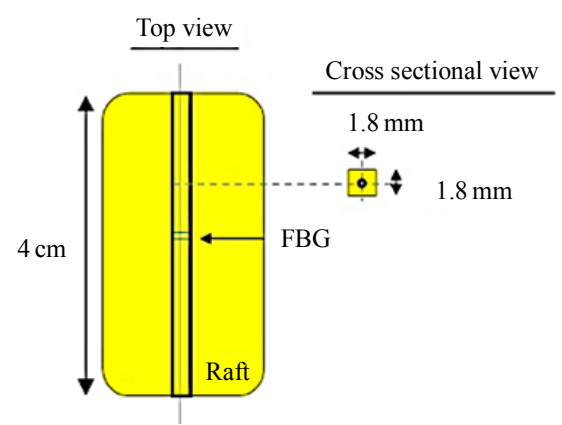

Fig. 1 Illustration (showing key dimensions) of the embedded FBG-based strain sensor designed in this work. 
super glue to ensure the sensor was operating to give optimum performance. The pre-stressed process of the FBGs was achieved by pulling the end of each FBG before the gluing process was carried out to ensure that the FBGs are in a tightly strained position.

\subsection{Sensing principle using this design of FBG-based strain sensor}

The FBG, which forms the basis of the sensor, is fundamentally a wavelength-selective filter that has been created in the usual way by exposing an optical fiber to an ultraviolet (UV) interference pattern, which then constructs a periodic change in the refractive index of the fiber core. Thus, a light that has a central wavelength corresponding to the Bragg condition will be reflected by the FBG sensor, as given by (1).

$$
\lambda_{b}=2 n_{\text {eff }} \Lambda
$$

where $\lambda_{b}$ is the Bragg wavelength that will be back-reflected, $n_{\text {eff }}$ is the effective index of the fiber, and $\Lambda$ is the grating period.

When the FBG is strained, the Bragg wavelength changes through the expansion or contraction of the grating periodicity, causing $\Lambda$ to increase or decrease respectively. Any variation in the surrounding temperature also affects the Bragg wavelength (due to the thermal expansion or contraction of the grating periodicity). In most practical applications, the Bragg wavelength of the FBG gives a shift $(\Delta \lambda)$ based on the combined strain $(\varepsilon)$ and temperature effect $(\Delta T)$, can be expressed as

$$
\frac{\Delta \lambda_{b}}{\lambda_{b}}=\left(1-p_{\text {eff }}\right) \Delta \varepsilon+(\alpha+\xi) \Delta T
$$

where $p_{\text {eff }}$ represents the photo-elastic parameter, $\varepsilon$ represents the change in the strain, $\alpha$ is the coefficient of thermal expansion of the fiber material, and $\xi$ is the thermo-optic coefficient of the fiber and describes temperature changes.

The FBG-based strain sensor developed in this work has been attached to a rigid polyvinyl chloride (PVC) pipe, as shown in Fig. 2. The sensors were placed at intervals, denoted by $h$, from each other to allow the device to measure strain at different positions on the pipe. The arrangement of the FBG sensors can be represented using the classical beam theory. The relationship between the curvature of the PVC pipe and the applied moment can be shown by (3) [27].

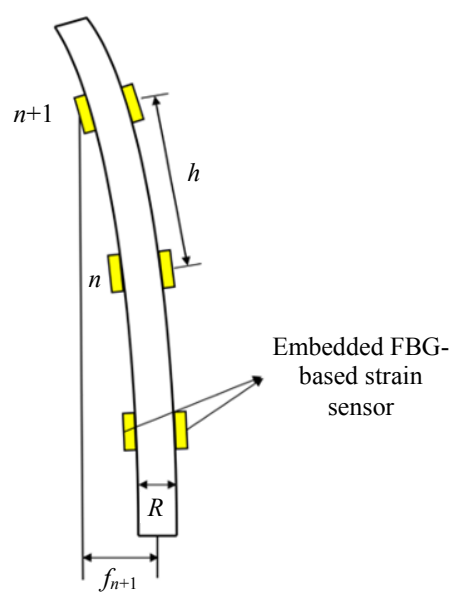

Fig. 2 Schematic of the working principle of embedded FBG-based strain sensing on the PVC pipe.

$$
k=\frac{M}{E l}=\frac{1}{\rho}
$$

where $k$ represents the curvature of the PVC pipe, $M$ is the pure bending moment applied to the PVC pipe, $E$ is the Young's modulus of the PVC pipe, $l$ is the moment of inertia, and $\rho$ is the rotational radius of curvature. The bending of the PVC along the horizontal axis can be simplified, and an equation representing its behavior is shown as $[27,28]$ :

$$
\Delta f=\frac{f_{n+1}-f_{n}}{h}
$$

where $f_{n}$ is the deflection at the $n$th FBG sensor position, $f_{n+1}$ is the deflection at the $(n+1)$ th FBG sensor position on the PVC pipe, and $h$ is the intervals between the embedded FBG-based strain sensors. The relationship between the deflections along the PVC pipe and the strains measured by the FBG sensors can be simplified and are indicated by (5) and (6) [27-29].

$$
\varepsilon_{n}=\frac{M R}{E l}=\frac{1}{h}\left[\frac{\left(f_{n+1}-f_{n}\right)}{h}-\frac{\left(f_{n}-f_{n-1}\right)}{h}\right]
$$




$$
\varepsilon_{n}=\frac{M R}{E l}=\frac{1}{h^{2}}\left(f_{n+1}-2 f_{n}-f_{n-1}\right)
$$

where $\varepsilon_{n}$ represents the strain measured by the $n$th FBG and $R$ the internal diameter of the PVC pipe. In addition, an additional FBG is usually included to provide a temperature-compensation mechanism to allow compensation for any temperature change cross-sensitivity in the measurements of the strain.

\section{Laboratory-based calibration tests}

\subsection{Comparison between bare and embedded FBG}

The experimental setup used in this work is illustrated in Fig. 3. First, a bare FBG was attached to the PVC pipe to obtain the initial spectrum of the unperturbed pipe, and then its wavelength shifted when the free end of the pipe was displaced at intervals of $2.5 \mathrm{~cm}$. These measurement steps were then repeated by embedding the same bare FBG in a 3D-printed TPU material (with dimensions stated in Fig. 1) to obtain the same type of measurements. This approach establishes the responsivity of the FBGs forming the sensor system, thus distinguishing the difference in performance between the bare FBGs and embedded FBGs used.

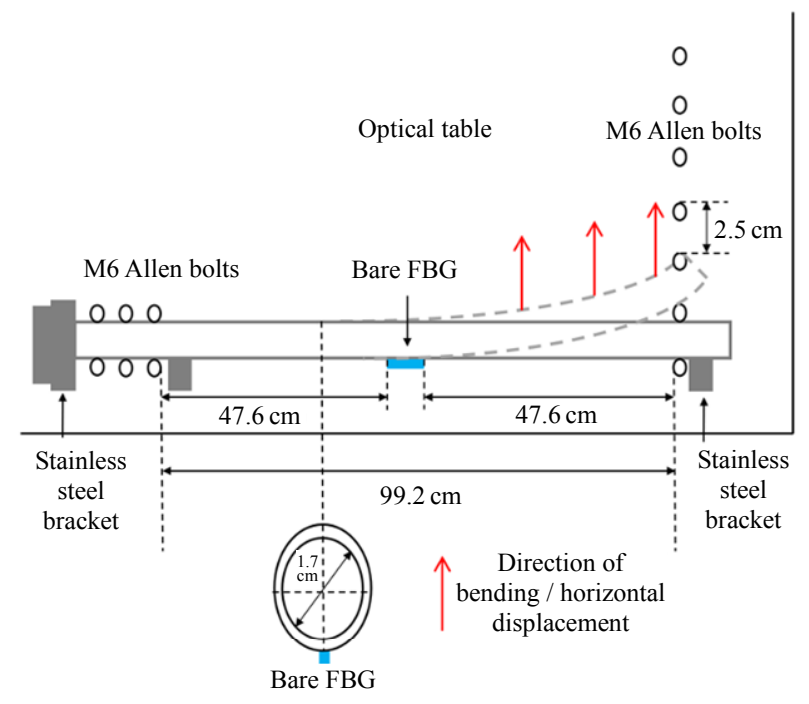

Fig. 3 Experimental setup for the bare and embedded FBG-based strain sensor measurements.

\subsection{Setup for multiple point measurements}

The experimental setup to evaluate the
FBG-based strain sensor can be seen in Fig. 4(a), showing that the strain variation relates to the horizontal displacement of the PVC pipe along with the optical table. First, a PVC pipe (with dimensions as stated in the figure) was laid horizontally on the optical table (equipped with a rectangular grid of tapped M6 holes). Following that, one end of the PVC pipe was fixed with a stainless-steel bracket and six identical (M6-type) Allen bolts (with three on each side) to prevent the PVC pipe from rotating during the experiment were carried out. Six FBGs (each of different central wavelengths and labelled as FBG 1 to FBG 6) were used and incorporated to form the basis of six different strain sensors, while an additional FBG (FBG T) was used for temperature compensation of the sensor system. The FBGs were then evenly distributed on the outer layer of the PVC pipe to allow the sensors to be used to evaluate the variation of the strains seen on the horizontal axis. Of the FBGs used, FBG 1, FBG 3, FBG 5, and FBG $T$ were spliced and placed on the tensile side of the pipe (with FBG $\mathrm{T}$ being loose inside the pipe to inhibit strain detection), whereas FBG 2, FBG 4, and FBG 6 were located on the compressive side of the pipe, after being spliced.

Figure 4(b) illustrates the schematic of the interrogation system. The response of the FBGs used was observed using a Yokogawa AQ6370C $(600 \mathrm{~nm}-1700 \mathrm{~nm})$ optical spectral analyzer (OSA), which was connected to Port 3 of the 3-port circulator used. An amplified spontaneous emission (ASE) light source was used and connected to Port 1, while the FBGs were connected to Port 2 via an optical switch, in which only 2 channels were used (positive $x$ and negative $x$ ). Table 1 shows the FBGs that were used in this experiment and their respective central wavelengths. The response and performance of the FBGs for strain measurement were evaluated based on the horizontal displacement of the free end of the pipe on the optical table. The bending direction and the strain sensors' position are shown in Fig. 4(a). When the PVC pipe was bent 
towards the compressive side of the pipe, FBG 1, FBG 3, and FBG 5 were found to experience tensile strain, while FBG 2, FBG 4, and FBG 6 were affected in the opposite direction (due to compressive strain), and vice versa (with FBG $T$ remaining unaffected by any strain changes, as designed). The response of the FBG was measured in the range of $0 \mathrm{~cm}$ to $15 \mathrm{~cm}$, using $2.5 \mathrm{~cm}$ intervals. The strain and stress of the pipe have a linear correlation, obeying Hooke's law (but only until the yield point, which can be determined by the offset method). This means that any displacements that exceed the yield point will cause the pipe to be permanently deformed (and it is not allowed to return to its original position).

Table 1 FBGs and their respective central wavelengths.

\begin{tabular}{cccc}
\hline FBG & $\begin{array}{c}\text { Centre } \\
\text { wavelength }(\mathrm{nm})\end{array}$ & $\begin{array}{c}\text { Position from fixed } \\
\text { end of pipe }(\mathrm{cm})\end{array}$ & $\begin{array}{c}\text { Condition } \\
\text { (Strain monitored) }\end{array}$ \\
\hline 1 & 1551.5 & 24.8 & Tensile \\
2 & 1551.5 & 24.8 & Compressive \\
3 & 1546.0 & 49.6 & Tensile \\
4 & 1546.0 & 49.6 & Compressive \\
5 & 1543.0 & 74.4 & Tensile \\
6 & 1543.0 & 74.4 & Compressive \\
T & 1540.0 & Inside the pipe & Loose \\
\hline
\end{tabular}

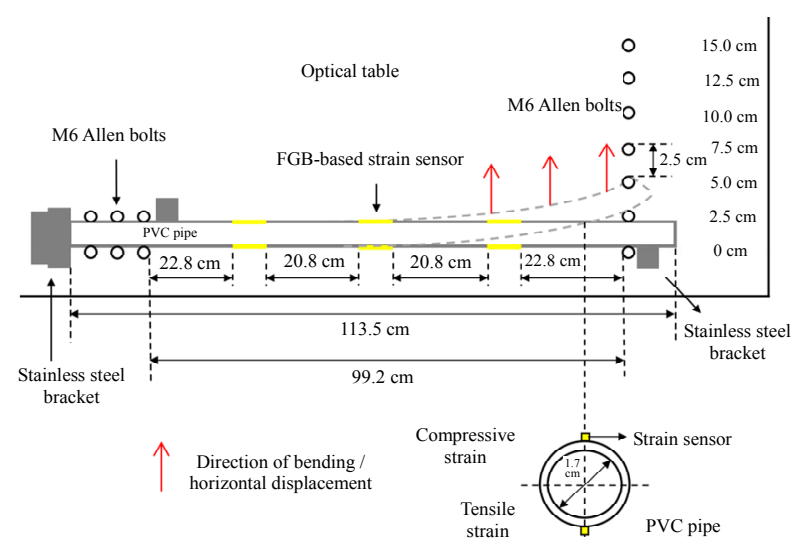

(a)

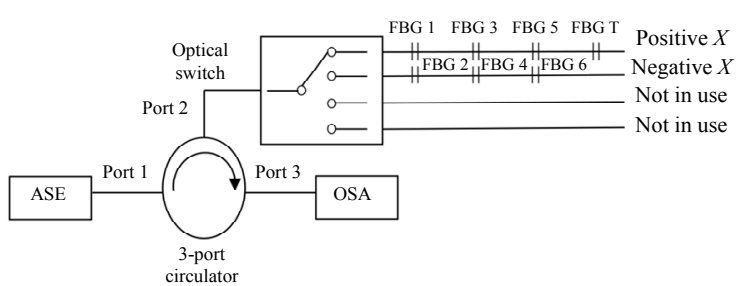

(b)

Fig. 4 Illustration of (a) the experimental setup of the embedded FBG-based strain sensor and (b) schematic diagram of interrogation system for the FBGs used (FBG 1 to FBG 6) and FBG T.
However, it was also important to compare the sensing performance of the embedded FBG-based sensor when it was embedded inside other materials such as PLA. This was to ensure and validate that TPU was more suitable as a sensing pad for the FBGs. So, an additional experiment has been carried out to compare the sensing performances between the FBG sensor embedded in TPU with other materials, which was PLA, as that was the only filament type available in our laboratory currently. A PLA-based strain sensor was fabricated, and the steps taken during the experiment were done in a very similar manner as in the case of the TPU-based strain sensor. The PLA-based strain sensor was placed exactly at the original position of FBG 5, $74.4 \mathrm{~cm}$ from the fixed end of the pipe.

\subsection{Temperature characterization}

The monitoring system illustrated in Fig. 4 had been designed with integral temperature characterization, where a series of measurements was carried out by placing an FBG sensor (labelled FBG T) inside a water bath to be calibrated, as illustrated in Fig. 5(c). First, a bare FBG T was placed

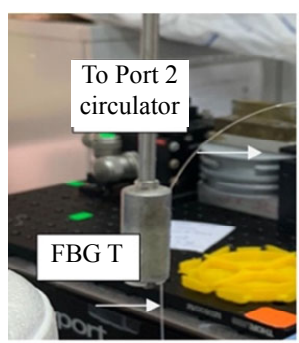

(a)

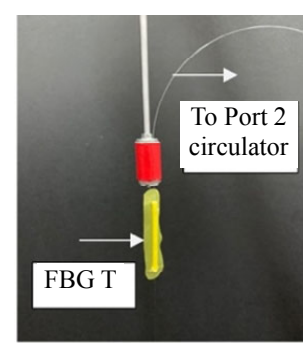

(b)

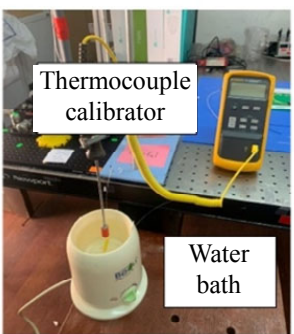

(c)

Fig. 5 Illustration of (a) bare and (b) embedded FBGs placement on thermocouple probe, and (c) the setup for temperature characterization experiment. 
vertically at the thermocouple probe, as shown in Fig. 5(a), to monitor the temperature inside the water bath. Then, to undertake the calibration, the temperature inside the water bath was set initially at room temperature $\left(25^{\circ} \mathrm{C}\right)$ and was increased gradually (in $5^{\circ} \mathrm{C}$ intervals) over the range from $25{ }^{\circ} \mathrm{C}$ to $50{ }^{\circ} \mathrm{C}$, while at the same time, the wavelength shift of FBG $\mathrm{T}$ was observed and the data were recorded. This was repeated for the embedded FBG T [where the placement of the FBG can be seen in Fig. 5(b)], so a comparison of performance could be made between the bare and the embedded FBGs, in terms of their temperature response.

\section{Results and discussion}

\subsection{Comparison of performance between bare and embedded FBGs}

Figure 6(a) shows the reflection spectrum of the FBG used before and after it was embedded inside the TPU material. A slight spectral wavelength shift $(0.136 \mathrm{~nm})$ could be observed when the FBG was embedded inside the thermoplastic (TPU) material, which could have been due to the pre-stressing of the FBG $(\sim 1.12 \mu \varepsilon)$ during the embedment process inside the material. The performance of both the bare and the embedded FBGs to the strain change seen was investigated, and the results are shown in Fig. 6(b). It can be observed that the sensitivity of the embedded FBG is $\sim 1.74$ times higher than that of the bare FBG. Figure 6(c) illustrates the sensing performance comparison between the TPU-based and the PLA-based strain sensor. From the figure, the sensitivity of the TPU-based is $0.0121 \mathrm{~nm} / \mathrm{cm}$, which is higher than that of the PLA-based device by $\sim 2.05$ times, having a value of $0.0059 \mathrm{~nm} / \mathrm{cm}$. This is due to the elasticity of the TPU, which is greater than PLA, due to its lower Young's modulus value. TPU has Young's modulus value of $6 \mathrm{MPa}$ [30], while the case of PLA has a value of $4.1 \mathrm{GPa}$ [31], which is significantly higher. A material with a higher Young's modulus value is considered less elastic (brittle) than a material with a lower value (ductile). Brittle materials tend to be very strong because they can withstand a lot of stress, cannot stretch, and break suddenly, while ductile materials are more elastic because they have a larger elastic region. Hence, the TPU-based casing would induce a larger strain to the FBG (larger wavelength shifts)

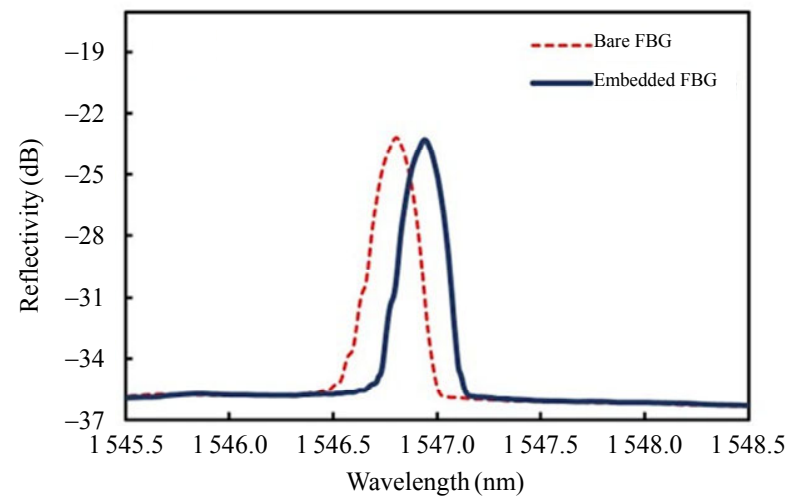

(a)

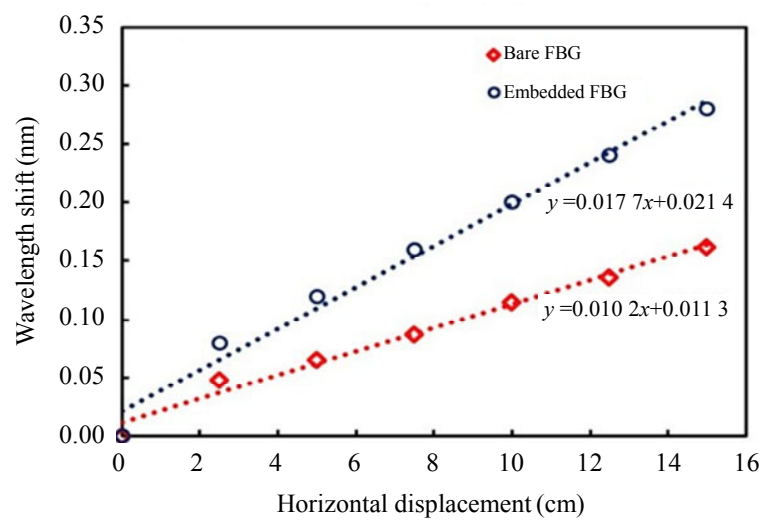

(b)

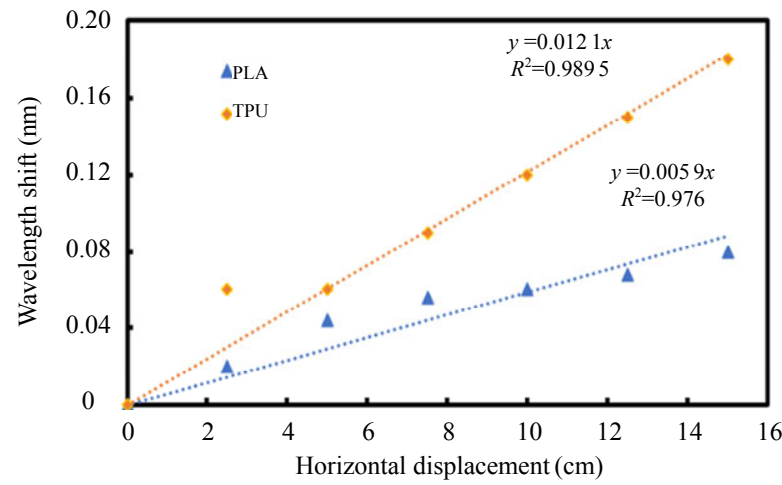

(c)

Fig. 6 Illustration of the spectra of (a) the bare FBG (dashed, red) and the embedded FBG (solid, blue), (b) graph of the calibration of both the bare (red) and embedded (blue) FBGs (as seen in Fig. 3), and (c) comparison of sensing performance between TPU-based strain sensor and PLA-based strain sensor. 
than the PLA-based casing when the same amount of force is applied. This proved that embedding the FBG inside TPU can be made reliable to provide a "sensing pad" for the FBG compared to PLA, which is very rigid and prone to breakage, especially in circular structures. Additionally, due to the elastic properties of the TPU material used, the sensitivity of the FBG to the bending effect of the pipe is enhanced, and this method also provides extra protection from external forces, which could damage or break the FBG (and which would otherwise be just a bare, exposed FBG).

\subsection{Multiple point measurements}

Table 2 shows the evaluation of each FBG (FBG 1 to FBG 6) with the horizontal displacement and its $R^{2}$ values for the linear fit. The graphs obtained as in Fig. 7 show a good linear response of the wavelength shift, with an average responsivity of $0.0135 \mathrm{~nm} / \mathrm{cm}$ to displacement for the tensile strain and $-0.0142 \mathrm{~nm} / \mathrm{cm}$ of displacement for the compressive strain. Each FBG shows a different degree of wavelength shift due to the different positions of the FBGs shown in the figures, reflecting that they react to the different levels of

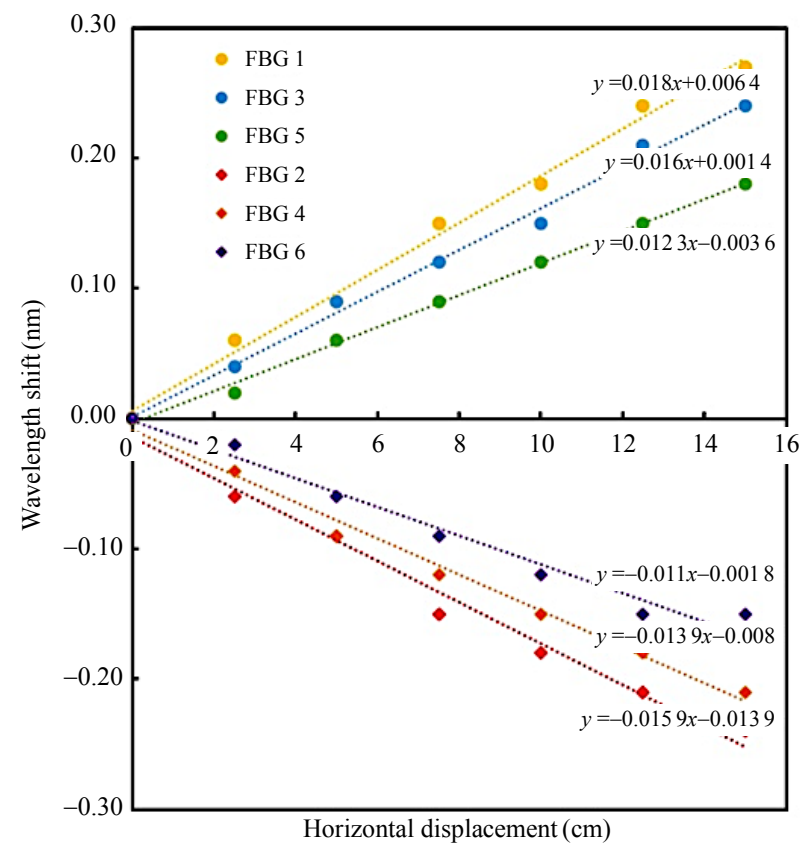

Fig. 7 Wavelength shift (nm) of the FBGs experiencing tensile (FBG 1, FBG 3, and FBG 5) and compressive strain (FBG 2, FBG 4, and FBG 6), in response to increasing horizontal displacement $(\mathrm{cm})$. induced strain experienced along the pipe. Apart from that, a very satisfactory linear fit is seen, and presents a good linear association with average $R^{2}$ values of 0.9770 for the tensile strain and 0.9764 for the compressive strain, creating a system that is easy to use in practice.

Table 2 Responsivity of each strain sensitive FBG to strain variation.

\begin{tabular}{cccc}
\hline FBG & Condition & Responsivity $(\mathrm{nm} / \mathrm{cm})$ & $R^{2}$ \\
\hline 1 & Tensile & 0.0180 & 0.9901 \\
2 & Compressive & -0.0159 & 0.9865 \\
3 & Tensile & 0.0160 & 0.9557 \\
4 & Compressive & -0.0139 & 0.9713 \\
5 & Tensile & 0.0123 & 0.9853 \\
6 & Compressive & -0.0111 & 0.9713 \\
\hline
\end{tabular}

\subsection{Temperature characterization}

The temperature sensitivities of the bare and embedded FBGs used are shown in Fig. 8, where the temperature sensitivities of the bare and embedded FBGs were monitored to be $11 \mathrm{pm} /{ }^{\circ} \mathrm{C}$ and $19.2 \mathrm{pm} /{ }^{\circ} \mathrm{C}$, respectively. An increase in the temperature sensitivity of the embedded FBG, as compared to bare FBG, was due simply to the larger thermal expansion of the TPU filament, which induces the strain on the FBG.

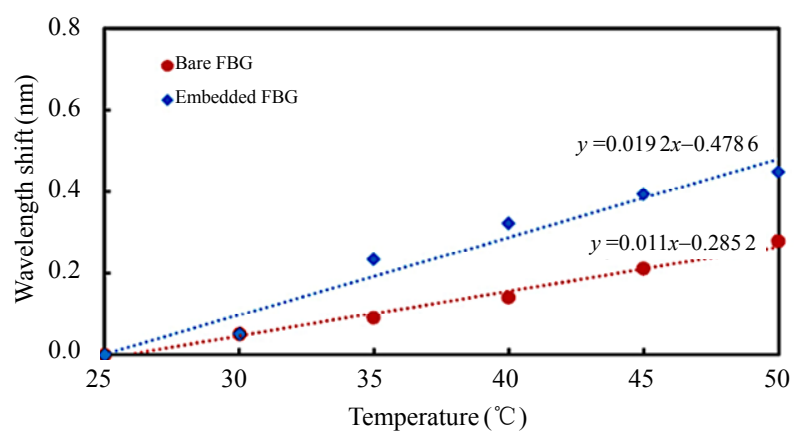

Fig. 8 Temperature sensitivity of bare (red) and embedded (blue) FBGs.

Considering the temperature compensation needed, for this reason, another embedded FBG (denoted as FBG T) was added to the system, and loosely placed inside the pipe [shown in Fig. 4(a)] to ensure strain insensitivity (as it is outside the strained region). The responses of the FBG $T$ to different horizontal displacements of the system and its sensitivity are shown in Figs. 9(a) and 9(c), respectively, illustrating its insensitivity to strain changes as the wavelength shift is not clearly seen in Fig. 9(a). Figure 9(b) illustrates a clearer figure 
showing the shift of wavelength of horizontal displacement for the cases of FBG 5 and FBG 3 on a magnified scale. The wavelength shifts against horizontal displacements for FBG 1, FBG 3, FBG 5, and FBG $\mathrm{T}$ are shown in Fig. 9(c), thus proving the FBG $\mathrm{T}$ is suitable as a temperature sensor to provide compensation as it had shown no response to strain changes.

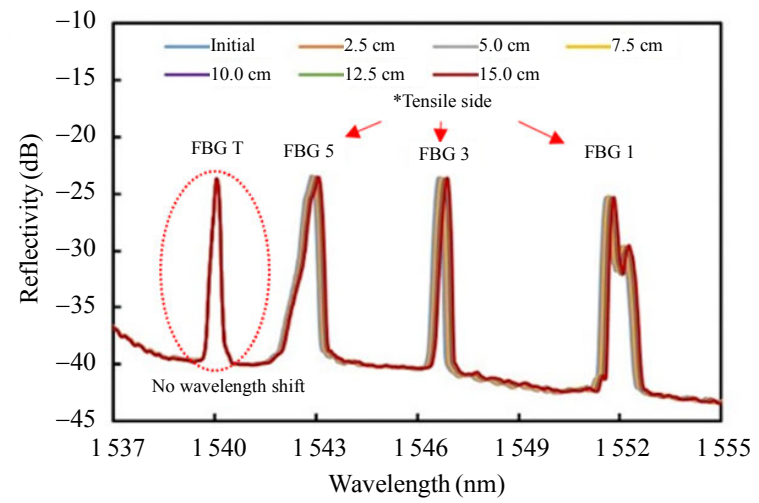

(a)

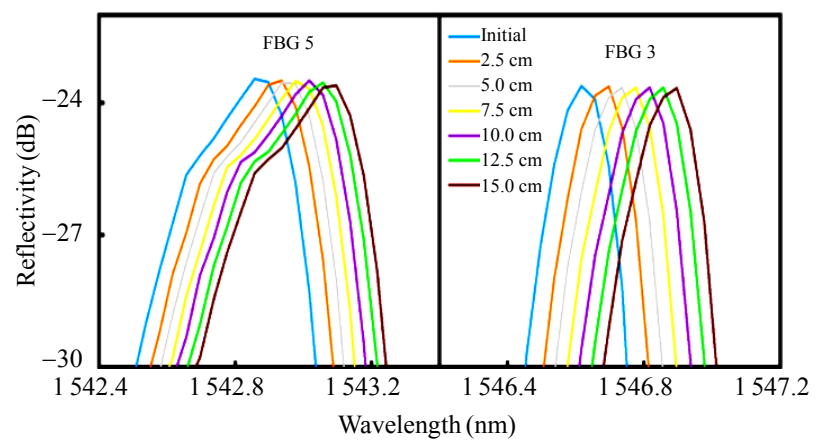

(b)

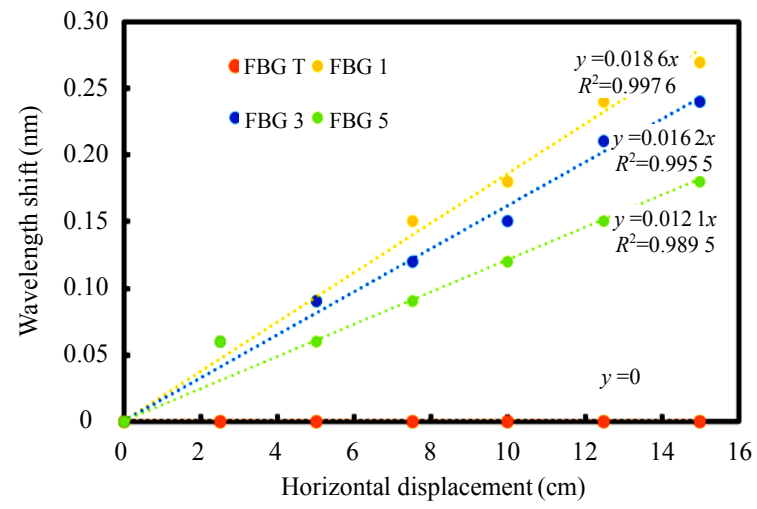

(c)

Fig. 9 Illustration of the (a) wavelength spectra of FBG 1, FBG 3, FBG 5, and FBG $\mathrm{T}$ at the different horizontal displacements shown $(0 \mathrm{~cm}$ to $15 \mathrm{~cm})$, (b) magnified wavelength shifts for FBG 5 and FBG 3, and (c) wavelength shifts (nm) of FBG 1, FBG 3, FBG 5, and FBG T during strain measurements over the same horizontal displacement from $0 \mathrm{~cm}$ to $15 \mathrm{~cm}$.

\section{Conclusions}

In this work, it has been demonstrated that the embedded FBG sensors inside the TPU, created by using an inexpensive 3D-printing technique, can be used effectively in measuring strain with high accuracy. The data obtained show an average responsivity $0.0135 \mathrm{~nm} / \mathrm{cm}$ for tensile strain and $-0.0142 \mathrm{~nm} / \mathrm{cm}$ for compressive strain, with high linearity of up to $99 \%$. Furthermore, temperature characterization of the system shows a linear correlation between the wavelength shift and the temperature, with a responsivity of $19.2 \mathrm{pm} /{ }^{\circ} \mathrm{C}$ for temperatures ranging from $25{ }^{\circ} \mathrm{C}$ to $50{ }^{\circ} \mathrm{C}$. Studies show that the embedded FBG has a strain sensitivity of $\sim 1.74$ times higher than that of the bare FBG. It was also seen that the TPU-based FBG strain sensor has a better sensitivity value of $\sim 2.05$ times higher than that of the PLA-based FBG strain sensor indicating that TPU was more suitable as a 'sensing pad' for the FBG.

\section{Acknowledgment}

This work was financially supported by a Newton Fund Impact Scheme under the NewtonUngku Omar Fund Partnership (Grant No. IF0222020). The grant is funded by the UK Department for Business, Energy and Industrial Strategy and Malaysian Industry-Government Group for High Technology (MIGHT) and delivered by the British Council and MIGHT. The authors are also pleased to acknowledge the support from University of Malaya (Grant Nos. RK021-2019 and TOP100PRC). Grattan also acknowledges the support from the Royal Academy of Engineering.

Open Access This article is distributed under the terms of the Creative Commons Attribution 4.0 International License (http://creativecommons.org/ licenses/by/4.0/), which permits unrestricted use, distribution, and reproduction in any medium, provided you give appropriate credit to the original author(s) and the source, provide a link to the Creative Commons license, and indicate if changes were made. 


\section{References}

[1] C. Hong, Y. Zhang, Z. Lu, and Z. Yin, "A FBG tilt sensor fabricated using 3D printing technique for monitoring ground movement," IEEE Sensors Journal, 2019, 19(15): 6392-6399.

[2] J. L. C. Diniz, R. D. Vieira, J. T. Castro, A. C. Benjamin, and J. L. F. Freire, "Stress and strain analysis of pipelines with localized metal loss," Experimental Mechanics, 2006, 46(6): 765-775.

[3] H. Xu, F. Li, W. Zhao, S. Wang, Y. Du, and C. Bian, "A high precision fiber Bragg grating inclination sensor for slope monitoring," Journal of Sensors, 2019, DOI: 10.1155/2019/1354029.

[4] H. H. Zhu, A. N. L. Ho, J. H. Yin, H. W. Sun, H. F. Pei, and C. Y. Hong, "An optical fibre monitoring system for evaluating the performance of a soil nailed slope," Smart Structures and Systems, 2012, 9(5): 393-410.

[5] J. Corominas, J. Moya, and A. Lloret, "Measurement of landslide displacements using a wire extensometer," Engineering Geology, 2000, 55(3): 149-166.

[6] L. Fang, T. Chen, R. Li, and S. Liu, "Application of embedded fiber Bragg grating (FBG) sensors in monitoring health to $3 \mathrm{D}$ printing structures," IEEE Sensors Journal, 2016, 16(17): 6604-6610.

[7] C. Y. Hong, Y. F. Zhang, M. X. Zhang, L. M. G. Leung, and L. Q. Liu, "Application of FBG sensors for geotechnical health monitoring, a review of sensor design, implementation methods and packaging techniques," Sensors and Actuators A: Physical, 2016, 244: 184-197.

[8] Y. Zhao and F. Ansari, "Quasi-distributed fiber-optic strain sensor: principle and experiment," Applied Optics, 2001, 40(19): 3176-3181.

[9] Q. Wang, J. Huang, Q. Liu, and Z. Zhou, "Dynamic strain measurement of hydraulic system pipeline using fibre Bragg grating sensors," Advances in Mechanical Engineering, 2016, 8(4): 1687814016645069.

[10] M. Maheshwari, Y. Yang, D. Upadrashta, E. S. Huang, and K. H. Goh, "Fiber Bragg grating (FBG) based magnetic extensometer for ground settlement monitoring," Sensors and Actuators A: Physical, 2019, 296: 132-144.

[11] C. E. Campanella, A. Cuccovillo, C. Campanella, A. Yurt, and V. M. N. Passaro, "Fibre Bragg Grating based strain sensors: review of technology and applications," Sensors, 2018, 18(9): 3115.

[12] M. C. Emmons, S. Karnani, S. Trono, K. P. Mohanchandra, W. L. Richards, and G. P. Carman, "Strain measurement validation of embedded fiber Bragg gratings," International Journal of Optomechatronics, 2010, 4(1): 22-33.

[13] S. W. Park, D. H. Kang, H. J. Bang, S. O. Park, and C. G. Kim, "Strain monitoring and damage detection of a filament wound composite pressure tank using embedded fiber Bragg grating sensors," Key Engineering Materials, 2006, 321-323(10): 182-185.

[14] D. S. B. A. Ismail, A. Kassim, H. B. Mohamad, A. S. A Rashid, and A. R. Bunawan, "Monitoring strain development of soil slope using distributed optical fibre sensor," IOP Conference Series: Materials Science and Engineering, 2019, 527(1): 012027.

[15] N. Tanaka, Y. Okabe, and N. Takeda, "Temperaturecompensated strain measurements using FBG sensors embedded in composite laminates," in SPIE's 9th Annual International Symposium on Smart Structures and Materials, San Diego, 2002, pp. 304-313.

[16] Y. C. Chen, C. C. Hsieh, and C. C. Lin, "Strain measurement for composite tubes using embedded, fiber Bragg grating sensor," Sensors and Actuators A: Physical, 2011, 167(1): 63-69.

[17] M. G. Zubel, K. Sugden, D. J. Webb, D. SáezRodríguez, K. Nielsen, and O. Bang, "Embedding silica and polymer fibre Bragg gratings (FBG) in plastic 3D-printed sensing patches," SPIE, 2016, 9886: $1-12$.

[18] A. G. Leal-Junior, C. Marques, M. R. N. Ribeiro, M. J. Pontes, and A. Frizera, "FBG-embedded 3-D printed ABS sensing pads: the impact of infill density on sensitivity and dynamic range in force sensors," IEEE Sensors Journal, 2018, 18(20): 8381-8388.

[19] C. Hong, Y. Zhang, and L. Borana, "Design, fabrication and testing of a 3D printed FBG pressure sensor," IEEE Access, 2019, 7(10): 38577-38583.

[20] P. C. Liacouras, G. T. Grant, K. Choudhry, G. F. Strouse, and Z. Ahmed, "Fiber Bragg gratings embedded in 3D-printed scaffolds," NCSLI Measure, 2015, 10(2): 50-52.

[21] S. F. Jiang, Z. H. Qiao, N. L. Li, J. B. Luo, S. Shen, W. $\mathrm{H}$. $\mathrm{Hu}$, et al., "Structural health monitoring system based on FBG sensing technique for Chinese ancient timber buildings," Sensors, 2020, 20(1): $1-17$.

[22] H. Sanada, Y. Sugita, and Y. Kashiwai, "Development of a multi-interval displacement sensor using fiber Bragg grating technology," International Journal of Rock Mechanics and Mining Sciences, 2012, 54: 27-36.

[23] J. Lee, Y. Kim, and J. H. Lee, "A 3-D-printed, temperature sensor based on mechanically-induced long period fibre gratings," Journal of Modern Optics, 2020, 67(5): 469-474.

[24] R. Liveira, R. Nogueira, and L. Bilro, "3D printed long period gratings and their applications as high sensitivity shear-strain and torsion sensors," Optics Express, 2021, 29(12): 17795-17814.

[25] M. Alsharari, B. Chen, and W. Shu, "3D-printing of highly stretchable and sensitive strain sensors using 
graphene based composites," in Eurosensors 2018 Conference, Austria, September 9-12, 2018, pp. 8-11.

[26] H. Tian, D. Liu, Y. Wang, and Q. Wang, "Effect of adhesive type on the sensitivity coefficient of FBG sensor bonded on the surface of CFRP," Optoelectronics Letters, 2019, 15(4): 264-268.

[27] Y. L. Wang, B. Shi, T. L. Zhang, H. H. Zhu, Q. Jie, and Q. Sun, "Introduction to an FBG-based inclinometer and its application to landslide monitoring," Journal of Civil Structural Health Monitoring, 2015, 5(5): 645-653.

[28] H. F. Pei, J. H. Yin, H. H. Zhu, C. Y. Hong, W. Jin, and $\mathrm{D}$. S. Xu, "Monitoring of lateral displacements of a slope using a series of special fibre Bragg grating-based in-place inclinometers," Measurement
Science and Technology, 2012, 23(2): 025007.

[29] H. Pei, J. Yin, and W. Jin, "Development of novel optical fiber sensors for measuring tilts and displacements of geotechnical structures," Measurement Science and Technology, 2013, 24(9): 095202.

[30] H. Lee, R. Eom, and Y. Lee, "Evaluation of the mechanical properties of porous thermoplastic polyurethane obtained by 3D printing for protective gear," Advances in Materials Science and Engineering, 2019, 2019: 5838361.

[31] V. C. Pinto, T. Ramos, S. Alves, J. Xavier, P. Tavares, P. M. G. P. Moreir, et al., "Comparative failure analysis of PLA , PLA/GNP and PLA/CNT-COOH biodegradable nanocomposites thin films," Procedia Engineering, 2015, 114: 635-642. 